\title{
Urgences
}

\section{Hamlet dans Mouchoir de nuages de Tristan Tzara}

\section{Martine Lévesque}

Numéro 25, octobre 1989

Multiples de Hamlet

URI : https://id.erudit.org/iderudit/025542ar

DOI : https://doi.org/10.7202/025542ar

Aller au sommaire du numéro

Éditeur(s)

Urgences

ISSN

0226-9554 (imprimé)

1927-3924 (numérique)

Découvrir la revue

Citer cet article

Lévesque, M. (1989). Hamlet dans Mouchoir de nuages de Tristan Tzara.

Urgences, (25), 30-40. https://doi.org/10.7202/025542ar d'utilisation que vous pouvez consulter en ligne.

https://apropos.erudit.org/fr/usagers/politique-dutilisation/ 


\title{
Hamlet dans Mouchoir de nuages de Tristan Tzara
}

Martine Lévesque

\begin{abstract}
On joue Hamlet. Cette représentation est une souricière et une surprise. C'est le Poète qui est et joue Hamlet. Vous me demanderez pourquoi; mais cela est le mystère du drame.
\end{abstract}

(Mouchoir de nuages, p. 340)

Le douzième acte de Mouchoir de nuages de Tristan Tzara est essentiellement composé d'extraits de Hamlet. Le Poète convie le Banquier et sa femme à venir voir cette pièce de Shakespeare parce qu'il croit qu'ils y découvriront tout le mal qui le et les hante.

Trois scènes de Hamlet ont été accolées pour former la souricière où le Poète compte piéger les époux. Dans cet acte capital pour le déroulement de la pièce de Tzara, on retrouve des parties de la scène 1 de l'acte II, de la scène 2 de l'acte II et de la scène 2 de l'acte III de Hamlet. Les extraits ne mettent en scène que trois personnages. D'abord, Ophélia confie à son père que Hamlet, pris de folie, a fait irruption dans son cabinet; puis elle sort tandis que Hamlet entre en scène. Après avoir pris Polonius pour un marchand de poisson, Hamlet soutient qu'il se laisserait mourir sans regret. Polonius quitte la scène momentanément et Hamlet en profite pour raconter que des créatures coupables, assistant à une pièce de théâtre relatant des crimes semblables à ceux qu'elles avaient commis, avaient avoué sur le champ leurs forfaits. Polonius revient pour avertir Hamlet que sa mère veut le recevoir; Hamlet prétend alors qu'il voit dans les nuages des formes d'animaux; le père d'Ophélia acquiesce et quitte la scène. Dans un court monologue, Hamlet avoue qu'il est maintenant capable de commettre les actes les plus sanguinaires.

L'acte XII est suivi de commentaires sur ce que peut signifier la représentation d'extraits de Hamlet dans Mouchoir de nuages. Les commentateurs, conscience (métatextuelle et 
distanciatrice?) du drame, expliquent la présence de Hamlet, d'Ophélia et de Polonius en tenant des propos sur Hamlet, sa mère et le roi Claudius.

Quoique le commentaire ne fasse pas partie à proprement parler de la représentation de Hamlet, il y a quand même plusieurs intrusions de cette pièce dans ce que disent les commentateurs. L'un d'entre eux dit: «N'ayant pas le temps de chercher et aussi par économie, économie, le fantôme se confond avec Hamlet." ${ }^{1}$ Ce même économie, économie se retrouve dans la pièce de Shakespeare. Quand Hamlet raconte à son ami le mariage précipité de sa mère, il dit: «Économie! économie, Horatio! Les viandes cuites pour les funérailles ont été servies froides sur les tables du mariage.» ${ }^{2}$ En parlant du Poète, un autre commentateur ajoute: «Il voulait que l'hameçon de son mensonge prenne ainsi la carpe de la vérité.» ${ }^{3}$ Il renchérit plus loin: «C'est la seule explication qu'on puisse donner à l'hameçon, car il n'y en a pas d'autres, étant donné que les époux Banquier sont une famille fort convenable, carpe de la vérité...." ${ }^{4}$ Chez Shakespeare, une réplique est déjà porteuse de celles de Tzara; Polonius livre cette pensée à Reynaldo: "Vous voyez maintenant: la carpe de la vérité se prend à l'hameçon de vos mensonges...." 5

En lisant les deux pièces dans leur totalité, on constate qu'elles traitent des mêmes problèmes. Shakespeare montre comment le mensonge et la vérité peuvent être difficiles à cerner : ils s'entrecroisent parfois à un point tel qu'il devient impossible de les distinguer. Hamlet feint la folie et l'égarement bien que sa conscience soit des plus aiguisées. Le roi Claudius prend l'attitude d'un frère affligé et d'un père compatissant, et cela après avoir commis un fratricide et s'être emparé du trône de son neveu. Dans Hamlet, tout n'est que mensonge et dissimulation: on se cache derrière les tapisseries, on dit le contraire de sa pensée, on se confond en ruses et en stratagèmes. Le même phénomène est présent dans Mouchoir de nuages. Il n'est pas aussi évident que dans Hamlet, mais certaines répliques ont un effet de grossissement. À deux reprises,

\footnotetext{
1 Tristan Tzara, CEuvres complètes (sous la direction de Henri Béhar), Tome I, p. 345.

2 William Shakespeare, Hamlet, p. 265.

3 Tzara, op. cit., p. 344.

4 bid., p. 345.

5 W. Shakespeare, Hamlet, acte II, sc. 1.
} 
32

les commentateurs affirment ceci: "On pourrait placer ici un très joli problème d'ordre général: À quel point la vérité est vraie. À quel point le mensonge est faux. À quel point la vérité est fausse. A quel point le mensonge est vrai. " ${ }^{6} \mathrm{Si}$ on prend la peine de préciser que la vérité est fausse et que le mensonge est vrai, c'est peut-être parce qu'il y a supercherie. Tout n'est peut-être pas aussi limpide que le laissent croire à d'autres moments les commentateurs.

Un regard panoramique jeté sur le déroulement des deux pièces révèle que le drame de Mouchoir de nuages gravite autour du même litige que celui de Hamlet. Dans les deux cas, un mariage oblige le héros à refouler un amour inavouable. Le Poète s'est fait ravir Andrée; depuis qu'ils s'étaient rencontrés, ils ne se quittaient que pour dormir. Sans être conscient de son bonheur, le Poète vivait avec elle des moments idylliques, lorsque le Banquier, maintenant ruiné et amoureux, vint revendiquer ses droits d'époux. Cette situation apparait aussi inattendue que le mariage de la Reine et de Claudius, lequel veut ainsi s'emparer du trône. À l'instar d'Hamlet, le Poète ne pouvait que se soumettre. Sans accorder d'importance à ses sentiments, on lui a arraché d'autorité ce qu'il croyait à la fois légitimement et inconsciemment posséder. Le mariage ancien d'Andrée, comme celui, tout récent, de la Reine, gâche ce qui aurait pu naître.

Certes, la blessure du Poète ressemble à celle d'Hamlet, mais il n'y a pas que cela qui unit les personnages. Ce qui les lie bien davantage, c'est que le Poète est sous la férule de Hamlet. L'esprit tourmenté du héros élisabéthain a repris vie dans celui du Poète. Marcel Herrand souffre d'un dédoublement de la personnalité. Il s'identifie tellement à Hamlet qu'il en vient à se prendre pour lui. En fait, il est persuadé qu'il est réellement Hamlet: «Poussez consciemment la folie à l'excès, vous serez moins fou que les autres. " 7 Toutefois, il ne tient pas à ce que son secret soit connu. Douée d'une vive intuition, Andrée le découvre rapidement. Elle lui dit: "Vous voulez me faire croire que vous n'êtes vous-même qu'un simulacre. ${ }^{8}$ Le Poète ne répond pas, mais il s'empresse de se ressaisir et de faire dévier la conversation. Quelques instants

\footnotetext{
6 Tzara, op. cit., p. 336.

7 Ibid., p. 336

8 lbid., p. 350.
} 
plus tôt, il s'est presque laissé prendre au jeu des confidences. Il a avoué qu'il n'aimait ni les hommes, ni les femmes, qu'il n'aimait que l'amour, c'est-à-dire la poésie pure. C'est ainsi qu'il a failli révéler son véritable identité, car pour Hamlet «L'homme n'a pas de charme [....] et la femme non plus ...»9

Le monologue de l'acte IX ressemble tout à fait à ceux que Hamlet adressait à son auditoire du début du XVIIe siècle. Le poète déclame une longue tirade en prose et en vers, où ses sentiments sont enrubannés de formulations désuètes et difficiles. Il se désole de l'épreuve qu'il doit traverser et s'afflige de cet amour qui ne cesse de le persécuter. Le discours de Marcel Herrand est l'écho lointain de ceux de Hamlet. Il y a là la même emphase, le même cri du cœur, le même désarroi devant la profondeur insondable des sentiments.

Dans la pièce de Shakespeare, Hamlet décide de piéger la conscience du Roi en le menant à assister à une représentation théâtrale qui lui remémorera son crime. Le Piège de la Souris devait mettre au jour sa culpabilité et l'accuser irrémédiablement. Le Poète reprend l'idée de son maître: il attire Andrée et son ami à une représentation de Hamlet pour que la vérité leur apparaisse enfin. "C'est le Poète qui est et joue Hamlet. " ${ }^{10}$ Le Poète ne joue pas seulement Hamlet, il est Hamlet! Il est même d'abord et avant tout Hamlet. Le contrat intertextuel vient d'être signé. Le Poète n'agit pas comme Hamlet puisqu'il est lui-même Hamlet. Il refait les mêmes gestes qu'il a faits plus de trois siècles auparavant: il invite d'abord les époux au théâtre et il assassine ensuite son rival. "Était-ce un meurtre simple ou compliqué du drame de la jalousie? Hamlet aurait-il tué le Banquier? "11 Que cela soit simple ou compliqué, il s'agit toujours du drame de la jalousie! Le commentateur ne se demande pas si le Poète a tué le Banquier; sans hésiter, il impute le meurtre à Hamlet. Ces deux personnages n'en font assurément qu'un seul. D'ailleurs, depuis le début, Marcel Herrand n'a jamais été lui-même. Il jouait faux car il tentait de cacher sa véritable identité. Et pourtant, tout dans ses gestes révèle la présence de Hamlet; même quand "le fantôme se confond avec Hamlet" 12, le Poète

\footnotetext{
9 Shakespeare, op. cit., p. 294.

10 Tzara, op. cit., p. 340.

11 Ibid., p. 346.

12 lbid., p. 345.
} 
est encore Hamlet, puisque le Spectre n'est nul autre que Hamlet père.

Lors de son séjour dans l'île, le Poète devient follement amoureux d'Andrée. Il semble subjugé par elle, mais son amour est platonique. Il aime chastement et en silence. Jamais il ne déclare son amour, il le garde précieusement pour lui. Il est prisonnier d'un secret qu'il ne peut avouer. L'explosion de son amour donnera lieu à une froide crise de jalousie; pour lamour d'Andrée, il abattra sauvagement le Banquier.

Pour comprendre les motivations du Poète, il faut comparer Andrée aux femmes que Hamlet a aimées. L'héritier de la couronne du Danemark est sérieusement secoué par le complexe d'CEdipe. Malgré son âge, il est toujours en plein conflit œdipien. Après le décès de son père, Hamlet a cru qu'il pourrait devenir le mari de sa mère. À cause de l'intervention de Claudius, son rêve s'est évanoui. Hamlet ne peut accepter le mariage incestueux de la Reine; il a l'impression que Claudius lui a arraché sa mère. Tout ce qu'il a refoulé resurgit. Le nouveau mari de sa mère, comme jadis son père défunt, devient le rival qu'il faut écarter. L'amour que le Poète voue à André comporte certaines similitudes avec celui que Hamlet portait à sa mère. D'abord parce qu'Andrée et Gertrude sont mariées, et que de ce fait elles deviennent inaccessibles: l'engagement matrimonial constitue un interdit inviolable. Le mari se dresse constamment devant l'être aimé. Gertrude, libre un instant, épouse le frère de son époux décédé. Andrée donne au Poète l'illusion qu'elle est libre pour finalement renouer avec son mari. Le mariage de Gertrude et celui d'Andrée sont les premiers éléments qui les lient l'une à l'autre. Ensuite, il y a le fait qu'elles sont toutes deux silencieusement aimées lorsqu'un revirement subit fait avorter cet amour.

Le Poète aime Andrée comme on aime sa mère: il lui prodigue un amour chaste et respectueux. Il refuse d'aller plus loin, puisqu'elle appartient à un autre homme. Le même phénomène se produit dans l'amour qu'un fils porte à sa mère. Il peut l'aimer, l'adorer, mais il ne pourra jamais la posséder. Le père jouit d'une telle suprématie que le seul fait qu'il soit là suffit à protéger son épouse et à écarter les rivaux. Le Poète est contraint d'adopter envers Andrée la même attitude qu'un fils doit prendre envers sa mère.

Andrée est partagée entre le Poète et son mari, comme la Reine est divisée entre Hamlet et le roi Claudius. Elles sont 
soumises à un déchirement perpétuel. Elles veulent satisfaire leur douce attirance, un peu maternelle, un peu charnelle, envers le Poète et Hamlet, mais elles sont d'abord astreintes aux désirs de leurs époux. Elles subissent un tiraillement constant; elles penchent vers les uns, mais elles sont retenues par les autres.

Certes, l'image de la Reine et celle d'Andrée peuvent se superposer. Certains traits les rapprochent indéniablement. Leurs mariages se révèlent aussi malencontreux l'un que l'autre, et elles représentent toutes deux la mère, de façon réelle ou fictive. Elles sont partagées entre deux types d'amour et leurs maris périront de cette double attirance. Toutefois, Andrée ne répond pas qu'à l'image maternelle puisqu'elle possède tous les acpects de l'amante. L'envoûtement du Poète pour cette femme rappelle celui que Hamlet eut autrefois pour Ophélia. Le Poète vit avec Andrée un amour démesuré qui n'atteindra jamais son dénouement. Il devra se soustraire à sa passion pour exécuter sa vengeance. Comme Hamlet, il délaissera celle qu'il aime pour aller vers son destin.

Quoique la relation entre le Poète et Andrée semble le rappel de celle entre Hamlet et sa mère, une réplique d'Andrée installe le doute. Elle dit au Poète: "Il me semble que j'ai toujours vécu dans le sang, la volupté et... les fleurs. Vous êtes poète, vous me comprenez... ${ }^{13} \mathrm{De}$ toute évidence, Andrée vient de s'ophéliser! La fille de Polonius n'a-t-elle pas vécue dans le sang, la volupté et les fleurs? Le Poète lui répond: «Et vous ne vous doutez pas, Madame, à quel point vous dites la vérité, car si vous saviez [...] si vous saviez...»14 Effectivement, Andrée ne sait pas, mais le Poète sait: il confirme qu'Andrée n'est nulle autre qu'Ophélia. Cependant, Andrée ne se détache pas de la Reine, puisque cette dernière représente d'abord la mère et que cette image ne peut se dissoudre: Hamlet aime aussi Ophélia comme un reflet de sa mère. La Reine représente le grand amour qu'il ne peut atteindre, Ophélia est le substitut légal à cet amour inavouable, et elle subira les sarcasmes que Hamlet, s'il ne respectait les interdits du Spectre de son père, devrait adresser à sa mère.

Andrée correspond à l'incarnation de l'amour idéal. Elle est à la fois la mère et l'amante. Elle personnifie l'amour

13 Tzara, op. cit., p. 336.

14 Ibid. 
36

parfait parce qu'elle réunit tous les charmes féminins. Le Poète aime Andrée aussi excessivement que Hamlet aimait Ophélia. Il est aussi choqué par l'intrusion du mari d'Andrée que le fut Hamlet par celle du deuxième mari de sa mère. Hamlet partage son amour entre deux femmes qui n'en font en fait qu'une seule; le Poète réserve son amour à une seule femme, mais la personnalité de cette dernière prend dans son esprit deux aspects particuliers.

En s'immisçant dans le couple, le Banquier prend l'image du père; il est le mari de la femme aimée, donc une symbolisation de l'autorité paternelle. Le Poète le déteste et le considère comme un rival. Toutes les frustrations enfantines du partage de la mère remontent à la surface.

Le Banquier, comme le roi Claudius, empêche le Hamlet de Shakespeare, devenu pour un temps celui de Tzara, d'aimer la femme de ses rêves. Par son unique présence, il relègue le Poète au second plan. Le triangle formé par la Reine, Hamlet et le Roi est semblable à celui que forment Andrée, le Poète et le Banquier. Claudius, en tant que mari de Gertrude, force Hamlet à s'incliner devant les droits qui lui sont conférés. La même situation se répète dans Mouchoir de nuages: le Poète doit s'effacer devant le mari d'Andrée. Claudius et le Banquier sont les époux, mais aussi les pères, puisqu'ils s'imposent comme mari de la femme aimée. Le mari d'Andrée n'intègre pas seulement l'image du roi Claudius, mais celle de tous les pères. Il concrétise la puissance paternelle avec tout ce que cela comporte: époux de la mère, père des enfants, rival et oppresseur. De même que Hamlet, dans son amour, confond sa mère et Ophélia, il en viendra à confondre Claudius et Polonius. Il ne subsistera que l'image du père. C'est donc volontairement que Hamlet tue Polonius! Comme le souligne Ernest Jones, il savait très bien qu'il ne s'agissait pas du Roi, puisqu'il venait tout juste de le quitter. Il transperce Polonius de son arme parce qu'il représente le père. Il le tue, puis traîne son corps hors de la chambre de sa mère. Dans Mouchoir de nuages, on réserve le même traitement au cadavre de Jacques: "Les apaches assassinent le Banquier et traînent son corps hors de la scène. " ${ }^{15}$ Les circonstances entourant la mort de Polonius et celle du Banquier se ressemblent étrangement. Dans les deux 
cas, on a traîné les corps dans un autre endroit. Le Poète élimine le Banquier parce qu'il représente tout ce qu'il déteste chez le père. Hamlet a agi de la même façon; en supprimant Polonius, il manifeste toute sa haine pour la figure du père castrateur.

Andrée, le Poète et le Banquier sont les personnages principaux de Mouchoir de nuages. En outre, lors de la représentation de Hamlet, on retrouve Ophélia, Hamlet et Polonius; et dans le commentaire de l'acte XII, il est question de la Reine, de Hamlet et du roi Claudius. Neuf personnages en tout, ou plus précisément, trois groupes de trois personnages. Ces groupes ne sont pas fixés définitivement, les personnages peuvent se mouvoir pour rejoindre ceux avec qui ils ont le plus d'affinités. Ils en viennent à se regrouper autour de trois figures dominantes.

Le premier groupe (Hamlet, le Poète et... Hamlet) s'alimente à une source unique: les multiples personnalités de Hamlet se matérialisent sous trois visages différents. Il y a d'abord le Poète "qui est et joue Hamlet»16. Il joue le rôle d'une âme pacifique et rêveuse; il s'affuble des habits de l'amoureux mais il n'en demeure pas moins très attentif à ses desseins. Il souhaite la disparition du Banquier et il finira par réaliser ses désirs. Dans la représentation de Hamlet, on retrouve un Hamlet follement épris d'Ophélia. Il tient des discours incohérents, et il semble troublé par tout ce qui lui arrive. Dans le commentaire de l'acte XII, surgit enfin le véritable Hamlet, qui ne supporte pas le mariage de sa mère et qui projette de mettre fin à l'existence de son oncle. Les trois Hamlet ont en commun les interdits que leur impose l'image paternelle. Ils adoptent des comportements de fils envers leurs pères. Les substituts paternels dressent un barrage entre eux et les femmes qu'ils aiment. Tous souhaitent que cette domination disparaisse d'une façon ou d'une autre. Jones affirme que Hamlet tuera Polonius et Claudius parce qu'il veut se débarrasser des "deux hommes qui le séparent de ses femmes" 17 . Il semble que les trois personnages veulent s'approprier les femmes de leurs pères. Ils se caractérisent avant tout comme étant des fils amoureux qui doivent abolir les images de pères pour accéder à l'autonomie.

16 Tzara, op. cit., p. 340.

17 Ernest Jones, Hamlet et CEdipe, p. 84. 
38

Andrée, Ophélia et la Reine forment le deuxième groupe. Ces trois femmes n'en font en fait qu'une seule. Andrée est une réincarnation d'Ophélia; or, Hamlet aime aussi Ophélia à travers l'image maternelle. Dans son esprit, elle n'est qu'un double de sa mère, elle devient en quelque sorte la mère elle-même. Andrée est sous la tutelle d'Ophélia, mais elle demeure toujours très semblable à Gertrude. La présence de son mari recrée la situation que Hamlet a vécue. Le Poète est confronté au Banquier; ce dernier prend l'image du père, et de ce fait Andrée gagne un fils. Limage de la mère se détache nettement de ce groupe.

Le Banquier, le roi Claudius et Polonius constituent le dernier groupe. Le roi et Polonius ont en commun leur paternité. Bien que le Banquier ne soit pas réellement un père, il incarne l'image du père, puisqu'il s'introduit dans la relation que le Poète entretient avec Andrée. Cette situation l'amène à symboliser l'autorité paternelle. Aux yeux du Poète, il possède tous les attributs d'un père réel. Les trois personnages se superposent dans l'image paternelle. Ils correspondent en tous points à l'idée que l'on se fait du père dominant. En écartant les enfants de leurs mères, ils redonnent vie au complexe d'Edipe.

Les trois figures dominantes de Mouchoir de nuages sont celle du fils, de la mère et du père. L'intrigue qui se noue entre les personnages procède du conflit œdipien. Le fils rêve de posséder sa mère, mais le père l'en empêche; il développe donc une rivalité envers ce dernier, jusqu'à souhaiter sa disparition. Ce résumé rend compte de toute l'histoire du Hamlet de Shakespeare, en plus d'éclairer celle de Mouchoir de nuages. Le Poète (le fils) aimait Andrée (la mère), mais le Banquier (le père) se dressait entre eux. Le Poète décide de l'éliminer.

La représentation de Hamlet dans Mouchoir de nuages résume et interprète ce qui s'est produit antérieurement dans la pièce et annonce ce qui va se produire subséquemment. Le douzième acte est un miroir de l'œuvre; il fournit un nouvel éclairage à tout ce qui était demeuré jusque-là obscur.

Au commencement du douzième acte, on se le rappelle, Ophélia raconte à Polonius la visite que Hamlet lui a rendue. Ils en viennent à la conclusion que Hamlet est devenu fou à cause de son amour pour elle. On nous prévient que la folie peut s'emparer d'un cœur qu'on empêche d'aimer. Le Poète ne peut pas se laisser bercer par les sentiments amoureux qui l'envahissent: il doit refouler l'amour qu'il ressent. Le ma- 
riage d'Andrée l'exhorte à taire ses sentiments. Cette situation finira par ébranler son esprit, jusqu'à le faire chavirer totalement. Lorsqu'Ophélia et Polonius conviennent de la folie d'Hamlet, ils annoncent celle qui va emporter le Poète.

Le Hamlet shakespearien prévient Polonius et le met en garde: sa fille pourrait engendrer, et ces naissances pourraient être malencontreuses. Il lui dit aussi de ne pas la laisser se promener au soleil, qui brille parfois d'une telle ardeur qu'il dérange les esprits. Hamlet est persuadé que rien de bon ne viendra du sein d'Ophélia; à l'entendre, il semble qu'elle devrait s'abstenir de procréer. Avec le retour de Jacques, le soleil inonde la vie d'Andrée et deux enfants viennent même s'ajouter à leur bonheur. Cependant, le couple n'aurait peut-être pas dû avoir d'enfants; en quelque sorte, il aurait dû suivre les conseils du Hamlet shakespearien, qui avait prévenu Polonius que cela pourrait être désastreux. L'arrivée des enfants a peut-être déclenché la fureur du Poète en lui apportant la preuve qu'Andrée ne lui était pas fidèle.

Dans le Hamlet de Shakespeare, quand Polonius s'enquiert des désirs de Hamlet, ce dernier lui répond qu'il aimerait bien se retrouver dans son tombeau. Polonius lui demande s'il peut prendre congé de lui et Hamlet lui dit: "Vous ne sauriez, monsieur, rien prendre dont je fasse plus volontiers l'abandon, excepté ma vie, excepté ma vie." 18 Hamlet ne supporte plus ses tourments: «L'aveu poignant d'Hamlet à Polonius ("sinon de ma vie", etc.) ne peut être interprété que comme l'aveu du désir de mort.»19 Après cette réplique de Hamlet dans la pièce de Tzara, s'amorce le long processus qui mènera le Poète à s'enlever la vie. Quarante ans plus tard, quand le temps a coulé et que la comédienne jouant Ophélia est si vieille qu'elle a de la difficulté à monter les escaliers, le Poète se tue dans sa mansarde. Sa mort demeure presque inexplicable. Pourquoi a-t-il attendu si longtemps avant de faire ce geste? Voulait-il être sûr que sa vengeance était accomplie? Le Spectre avait ordonné à Hamlet de ne rien tenter contre la Reine: "que ton âme s'abstienne de tout projet hostile à ta mère! abandonne-la au ciel et à ces épines qui s'attachent à son sein pour la piquer et la déchirer. . 20

18 Shakespeare, op. cit., p. 291.

29 Jones, op. cit., p. 67.

20 Shakespeare, op. cit., pp. 276-277. 
40

Le Poète ne voulait pas mourir avant d'être assuré que le Banquier «allait agacer le souvenir qu'il laissa dans la vie d'Andrée" 21. Les souffrances de la femme du Banquier étaient destinées à satisfaire le Spectre: le père de Hamlet voulait que la Reine soit dévorée par les remords.

Lorsque Polonius prévient Hamlet que sa mère voudrait le rencontrer, celui-ci répond qu'il ira tout à l'heure. Il reprend ensuite pour lui-même: "Tout à l'heure est aisé à dire." $22 \mathrm{Il}$ n'a nullement l'intention d'aller retrouver la Reine, ni tout à l'heure, ni jamais: le Poète et Andrée se voient ce soir-là pour la dernière fois. Maintenant Hamlet pourrait «boire du sang chaud et faire des actions si amères que le jour frémirait à les regarder $» 23$.

L'acte XII contient tous les moments importants de Mouchoir de nuages: l'amour menant à la folie, le meurtre du Banquier, le suicide du Poète, les remords d'Andrée. Maintenant, il ne subsiste aucun doute: Hamlet a bel et bien joué l'un des plus grands rôles de sa vie dans Mouchoir de nuages!

En assemblant les personnages, nous avons découvert un lien très étroit unissant Hamlet et Mouchoir de nuages. Les deux pièces sont centrées sur le même conflit œdipien. Elles mettent en scène des personnages qui se ressemblent si étrangement qu'ils en viennent à se confondre; leurs particularités s'estompent au profit du mythe qu'ils illustrent.

Sophocle, Shakespeare et Tzara, à des époques éloignées, ont écrit sur le même sujet. La rivalité qui naît entre le fils et le père pour la possession de la mère n'a pas fini d'agiter l'âme des dramaturges. Elle est enfouie dans l'inconscient, et elle finit toujours par resurgir dans la demi-conscience de l'écriture.

\section{Bibliographie}

Jones, Ernest, Hamlet et CEdipe, Paris, Gallimard, 1967, 188 p.

Shakespeare, William, Richard III, Roméo et Juliette, Hamlet, Paris, GarnierFlammarion, $1979,373 \mathrm{p}$.

Tzara, Tristan, CEuvres complètes (sous la direction de Henri Behar), tome I, Paris, Flammarion, 1975, 746 p.

21 Tzara, op. cit., p. 350.

22 Ibid., p. 344.

23 lbid. 


\section{Daniel Schiff \\ Hamlet recyclé chez Cosinus}

$\mathrm{M}$

AZETTE ! ce n'est pas Schiff qu'il devrait s'ap peler, c'est Chiftre! Si vous êtes allergique aux "mathematiques séveres "du vieux Ducasse, alors lisez la Ligne de Sceaux, cette histoire ecrite par un dandy de l'hypotenuse qui va vous réconcilier pour toujours avec l'art de Pythagore... Car Daniel Schift a réussi ce petit exploit : faire du roman avec le jargon des Arts et Métiers, encanailler les sinus et les cosinus en les epinglant au veston de la tiction... Resultat ? Le voila : les maths, c'est la poesie moderne. Et c'est aussi terriblement drōle, mais oui, si l'on veut bien venir noyer un instant ses certitudes dans la baignoire d'Archinede, la ou, à côté du bon monsieur Que neau, nous attendent tous les logiciens du délire et autres maniaques du compas truque.

Marc, le heros de Daniel Schiff, est ce qu'on appelle une grosse tête : il fait dans la physique des metaux et transporte, entre deux stations de la ligne de Sceaux, une tosse des maths a laire pàlir Carabosse et Polichinelle reunis. Lorsque commence le roman, notre homme arrive douilettement a la quarantaine, mais on dirait qu'il se passe des choses bizarres dans sa petite caboche: il voit, le malheureux, des electrons partout, en particulier sous le tee-shirt des dames... Deformation professionnelle?
L'erotisme genre math-sup, quel pied, surtout lotsquion traque la mensuration a trois chiffres apres la virgule. Quant aux courbes de Gauss, r'est-ce pas, elles ne sont pes toutes la out l'on croyait..

\section{Un obsédé de la fesse}

Resumons donc on disant que notre héros est un obsede de la fesse qui a mis ses fantasmes en equations pour eviter le pire. Mais gare! Même de ce côtè-la, ça va degringoler, badaboum: Marc croit avoir écrit un article supersavant, la revelation du siecle, jusqu'au jour oú un colleggue lui tait remarquer qu'il a 'ait une grossiere erreur de signe qui fiche tout en l'air! Vacherie d'existence!'Dans son wagon de banlieve qui lui sert de tanière, le bougre sort donc sa petite serviette, et il recommence les calculs... Il cherche le pepin, biffe, jure, rature, enrage, s'emmels les pinceaux tandis que le rapide trépigne entre une avalanche de decimales et quelques poitrines de voyageuses transparentes comme un pub de chez Scandale. Bret. on a vite compris oú Daniel Schift voulait en venir : lenler de son matheux recale courant apres ses logarithmes bidons et ses tantas. mes de trustré, c'est nous! Nous. les trappeurs des certitudes a jamais perdues, nous qui essayons d'oublier nos vieilles frayeurs avec des balivernes de technocrates bernès...

Voila pour l'histoire. Mais ce qui est bizarre, vrainent tizarre, c'est que tout ça n'est pas dit avec des mots ordinaires, car il y a un "style Daniel Schiff " qui n'appartient qu'a lui: plus proche du tableau noir d'un labo que de la NRF, Ce que ça donne? Eh bien, a longucur de pages, on tornbe sur des hectomètres de calculs, de veritables batailles rangées de signes au milieu de brouettées de formules, et en avant la musique... Le plus fort, c'est qu'on marche! lmpression d'être sur le bloc-note de Maxwell, comme si ce bouquin avait gobe les choses de la vie pour les raconter avec les mots de nain. tenant : ceux qui jaillissent, bardes d'algebres mysterieuses, des com. puters et des supel-cerveaux. Et en plus, c'est follement drole, pince. sans-rire tant qu'on en veut! Voila ce qui s'appelle être de son temps. Car, a l'epoque d'jBM. Hamlet doit se recycler chez Cosinus. II the peut plus se contenter de pleurnicher dans son mouchoir. Finis les snifs-snils, il faut ecrire a theure du bip-bip. Daniel Schity l'a compris, il est le barde de demain.

André CLAVEL

\section{LA LIGNE DE SCEAUX}

do Daniel Schiff

(Minuit, 142 p., $39 \mathrm{~F}$ )

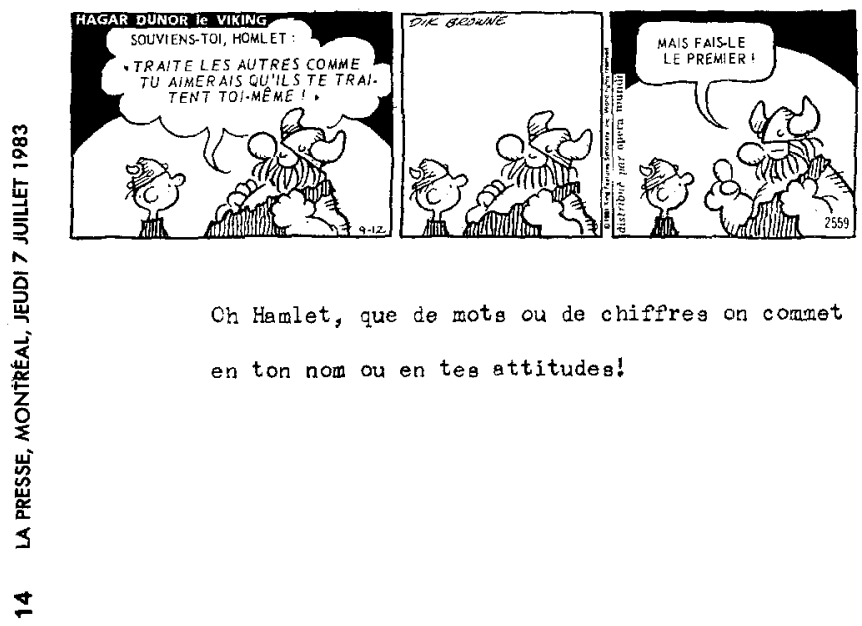

